\title{
Innovación y la Importancia de los Flujos Externos de Información: una Propuesta para el Análisis de los Contactos Informales
}

\author{
Alexandre Trigo'
}

\begin{abstract}
The number of empirical studies on informal contacts in the field of innovation is still very low due to the lack of variables able to identify and estimate the knowledge transfer through informal relationships among individuals. As a way to overcome this lack of measurement tools, we propose a analysis based on variables found in the Community Innovation Survey. The application of our methodology to a sample of service innovating firms, using the Spanish Technological Innovation Panel (PITEC) as data source led us to identify two groups of activities with a high propensity to establish informal contacts with customers and suppliers. This result has also allowed us to recognize external flows of information in low technology-intensive sectors; this evidence could hardly be observed through traditional indicators of cooperation due to the techno-scientific nature and formal, structured character of the partnership represented in these variables.
\end{abstract}

Keywords: Informal contacts; innovation; information flows; community innovation survey; cooperation.

\section{Resumen}

Los estudios empíricos sobre los contactos informales en el ámbito de la innovación son escasos debido a las limitaciones a la hora de construir indicadores capaces de identificar y estimar la magnitud de este tipo de intercambio de conocimiento entre individuos. Para poder solventar esta falta de instrumentos de medición, este artículo presenta como propuesta un análisis a partir de variables existentes en la Community Innovation Survey. Siguiendo esta metodología para una muestra de empresas de servicios en España extraída del Panel de Innovación Tecnológica (PITEC), se identificaron dos grupos de actividades con elevada propensión a establecer contactos informales con los clientes y proveedores. Este resultado ha permitido identificar flujos externos de información en sectores poco intensivos en tecnología que difícilmente podrían ser observados a través de indicadores tradicionales de cooperación debido al carácter tecno-científico de las alianzas representadas en estas variables.

Keywords: Contactos informales; innovación; flujos de información; community innovation survey; cooperación.

Grupo ICEDE, Departamento de Economía Aplicada, Universidad de Santiago de Compostela ,Av. Burgo das Nacións, s/n. I5782 Santiago de Compostela - España Teléfono: (34) 88I 8II 653. E-mail: alexandretrigo@gmail.com 


\section{Introducción}

Durante las últimas décadas, la literatura sobre innovación ha resaltado de forma creciente la importancia de los flujos externos de información (Rothwell \& Robertson, 1973; Rothwell, 1992, 1994; Lundvall, 1992; Gibbons et al. 1994; Oerlemans, Meeus \& Boekema, 1998; Chesbrough, 2003; Chesbrough, et al., 2006; Vega-Jurado, GutiérrezGracia, Fernández-de-Lucio, 2009) de tal manera que actualmente, las redes de relaciones y la interacción con otros agentes se han convertido en la base del proceso de innovación de muchas empresas. Dentro de este extenso campo de investigación, muchos autores han tratado de destacar la relevancia de las colaboraciones para la innovación en las empresas, incluyendo específicamente los contactos informales (Chandler, 1962; Dougherty, 1999; Dahl \& Pedersen, 2004; Cross \& Parker, 2004; Allen, James \& Gamlen, 2007; Taminiau, Smit \& de Lange, 2009). A pesar del reconocimiento de la importancia los contactos informales entre empresas y demás agentes para la innovación, el número de trabajos empíricos todavía es muy reducido debido a la dificultad de medir este tipo de relaciones y a las limitaciones a la hora de construir indicadores precisos capaces de identificar y estimar la magnitud de la transferencia de conocimiento a través de estas vías. En este sentido, este artículo pretende cubrir esta laguna ofreciendo un análisis que permite aproximarse al estudio de los contactos informales entre empresas y otros agentes.

La medición de la innovación siempre ha entrañado inúmeras dificultades, incluyendo el análisis de los flujos de información entre diferentes agentes del sistema. Para resolver en cierta medida la mayoría de esos problemas, la Organización para la Cooperación y el Desarrollo Económico (OCDE) realizó un importante esfuerzo en elaborar y mejorar indicadores para el control de la innovación y del cambio tecnológico en las empresas, no sólo desde un punto de vista empresarial sino también de una perspectiva de la política pública económica. El resultado de este esfuerzo se ha condensado en el conocido informe titulado "Manual de Oslo", donde se encuentran las directrices propuestas por la OCDE para la recogida e interpretación de datos sobre innovación tecnológica. Actualmente, este manual es el marco teórico para la elaboración de los cuestionarios utilizados en la Community Innovation Survey (CIS).

La CIS consiste en encuestas armonizadas que proporcionan información sobre la performance innovadora de di- ferentes sectores de diferentes países europeos. El cuestionario utilizado cubre una amplia gama de variables de innovación para una amplia muestra de empresas. Sin embargo, los indicadores sugeridos para medir las redes de relaciones y colaboraciones para la innovación se restringen a las cooperaciones formales, bajo contrato y acuerdo mutuo, y a las fuentes de información en general. A través del cruce de ambas variables, este artículo presenta una solución alternativa a la medición de los contactos informales. El estudio empírico está construido en base a los micro-datos que componen el Panel de Innovación Tecnológica (PITEC) para empresas de servicios en España, que a su vez, está creado a partir de la CIS española. Esta base de datos permitirá estudiar el alcance de los flujos de información para veinte actividades de servicios así como los diversos tipos de agentes con quienes la empresa puede establecer relaciones tal como, por ejemplo, proveedores, clientes, competidores y centros tecnológicos.

El artículo está estructurado de la siguiente forma. En el apartado 2, se presentan los fundamentos teóricos a partir de la concepción de la innovación como un proceso sistémico e interactivo, donde las relaciones y colaboraciones externas juegan un papel cada vez más determinante. Se introduce también en este apartado la problemática del análisis de los contactos informales en el ámbito de la innovación. La metodología y las fuentes de datos utilizados para el análisis empírico componen el apartado 3. El apartado 4 trata de presentar los resultados obtenidos tanto con respecto a la innovación en el sector servicios como a los contactos informales con diferentes agentes. El apartado 5 está dedicado a las principales conclusiones de este estudio.

\section{Fundamentos teóricos}

\section{I Los flujos externos de información}

El desempeño innovador de muchas empresas en las últimas décadas nos ha permitido contemplar como los procesos de innovación son cada vez más constantes y con distintas facetas, no sólo asociados a desarrollos y cambios puramente tecnológicos en sectores determinados. Al contrario, la innovación posee un inmenso abanico de elementos determinantes además de las tradicionales actividades internas de $\mathrm{I}+\mathrm{D}$, sobre todo cuando ampliamos el análisis a ciertos sectores económicos como por ejemplo 
los servicios. La tendencia creciente a la especialización y a la externalización de tareas y funciones empresariales han acentuado la importancia del entorno organizativo en los modelos actuales de gestión, en los que las fronteras empresariales se han hecho cada vez más permeables. Esta perspectiva contrasta en gran medida con los modelos canónicos y tradicionales basados en procesos cerrados de creación de conocimiento. Dicho eso, el estudio contemporáneo de la innovación y de su generación entraña la necesidad de buscar nuevos elementos distintos de la I+D interna y aislada, como pueden ser los puentes de conocimiento representados por las contactos, redes y alianzas entre empresas y otros agentes económicos. En la esfera de la investigación, diferentes estudios empíricos han puesto en evidencia que las empresas difícilmente innovan de forma aislada, apartadas del sistema (Christensen \& Lundvall, 2004).

Esta necesidad de analizar la empresa como un elemento del sistema de innovación, así como sus relaciones con los demás agentes económicos que componen dicho sistema pone de manifiesto, por lo tanto, el carácter dinámico, abierto e interactivo de la innovación y del aprendizaje. El argumento que sostiene este planteamiento analítico se basa en el hecho de que el conocimiento necesario para innovar se encuentra disperso por la sociedad, con lo que las fuentes de información para la innovación se encuentran en gran medida más allá de las fronteras de la empresa (Hayek, 1945). Los clientes, por ejemplo, son importantes fuentes de información - además de co-autores de innovaciones - así como los proveedores y las universidades, entre otros (Tether, 2002b). Resulta razonable afirmar, por lo tanto, que la capacidad innovadora de una empresa o de un sector económico crecerá en función de la capacidad de interacción con los demás agentes económicos.

Desde el punto de vista teórico, tanto el carácter colectivo de la innovación como el carácter compartido del aprendizaje han sido recogidos en distintos enfoques como los Sistemas de Innovación (Freeman, 1987a,b, 1995; Lundvall, 1992; Nelson, 1993; Edquist, 1997), el concepto de innovación como un proceso distribuido y disperso (Andersen, Metcalfe \&Tether, 2000; Coombs, Harvey \& Tether, 2003) así como la concepción de la creación de conocimiento como producto de interacciones localizadas entre un amplio rango de agentes heterogéneos, capaces de aprender y establecer relaciones en red (Antonelli, 1998, 2005). Recientemente, nuevos conceptos han sido introducidos en la literatura, como por ejemplo el para- digma Open Innovation (Chesbrough, 2003; Chesbrough et al., 2006) como forma de plasmar la práctica abierta y colectiva de la generación de conocimiento y, por lo tanto, de la innovación desarrollada por muchas empresas.

En el sector servicios en particular, el carácter participativo del proceso de innovación merece ser especialmente destacado debido a la propia naturaleza interactiva del proceso de producción, de prestación y de consumo de la mayor parte de sus actividades (Miles, 1993, 1994, Miles \& Tether, 2003, Gallouj, 2007). Esa naturaleza interactiva, intrínseca a la propia actividad, favorece la generación de las condiciones necesarias para la aparición de cooperación en las relaciones verticales, tanto con los proveedores como con los clientes, en el proceso de innovación.

\subsection{El estudio de los contactos informales en la innovación}

La gran mayoría de los estudios que trataron de analizar los flujos externos de información ha tenido como principal referencia los acuerdos formales de cooperación (Gemünden, Heydebreck \& Herden, 1992; Tether, 2002b; Czarnitzky \& Fier, 2003; Tödtling et al., 2009). No obstante, además de los acuerdos o alianzas formales en proyectos de innovación, otras vías desvinculadas de marcos contractuales juegan un papel capital en la generación y transmisión de información y conocimiento, tanto a nivel interno como externos a la empresa. Se trata de relaciones informales entre empresa y otros actores del sistema económico como los clientes, proveedores, colegas de profesión, entre otros, sin la preexistencia necesaria de contratos de mutua colaboración para la innovación.

Mucho se ha escrito sobre la importancia de los contactos informales, pero poco se ha discutido sobre la relevancia de las informaciones transferidas en la capacidad innovadora de las empresas (Dahl \& Pedersen, 2004). En este sentido, Dahl y Pedersen (2004) realizaron un trabajo empírico para medir el valor para la empresa de la información transmitida a través de contactos informales. Los resultados de la encuesta propia con empresas del norte de Dinamarca mostraron que la información transmitida poseía, en esencia, un carácter general en vez de técnico, con un grado de importancia mediana para la empresa. Taminiau, Smit y de Lange (2009), sin embargo, recalcan que los flujos externos de información, en particular a los contactos informales, son no solo una 
vía alternativa de transmisión de conocimiento, sino la forma más eficaz de optimizar la innovación para muchas empresas. Estos resultados confirman el carácter vital de este tipo de intercambio "desestructurado" para la gestión del conocimiento empresarial. El principal desafío de los modelos estratégicos de gestión empresarial, y en particular de gestión de la innovación, es crear un ambiente favorable, así como estructuras que permitan y faciliten este tipo de relaciones espontaneas (Dougherty, 1999).

Ahora bien, a la hora de medir y analizar intercambios de esta naturaleza, es necesario tener en consideración que los flujos informales de información son parte del día a día de las relaciones profesionales, y pueden estar expresados en dimensiones muy diversas. Por lo tanto, cualquier esfuerzo por medirlos seguirá siendo una mera aproximación. Los contactos informales pueden surgir a partir de diferentes tipos de actividades sociales: conferencias, ferias, así como contactos entre individuos de la misma empresa (o incluso de la competencia), antiguos empleados, clientes, proveedores, etc. Resulta importante resaltar además que los contactos informales también están presentes en contextos enmarcados por contratos formales de colaboración. En este sentido, los contactos formales e informales constituyen conjuntamente redes sociales de comunicación e intercambio de información y conocimiento.

Chandler (1962) introdujo el concepto de redes sociales (social networks) para describir las estructuras formales e informales de transmisión de conocimiento. Sobre este aspecto, Allen, James y Gamlen (2007, 19I) matizan que "the differences between formal structures and the informal organization are frequently attributed to the fact that although managers are aware of the importance of informal networks in the production and realization of knowledge (...) they fail to exploit them effectively as a mechanism for the exchange of knowledge assets". Estos autores trataron de analizar los flujos formales e informales de información en redes de I+D. Los resultados obtenidos, a partir de la aplicación de la herramienta social network analysis, demuestra que la configuración de ambos flujos no coinciden en absoluto. En otras palabras, las personas en las empresas no siguen necesariamente el modelo jerárquico ni las distribuciones oficiales de departamentos y funciones para realizar sus consultas (véase también Cross \& Parker, 2004). Esto pone en evidencia la existencia de individuos altamente activos en lo que respecta a la captación y transmisión de información en las empresas, cuya función en la organización no se co- rrespondería necesariamente con esta actividad. Este estudio también pone de manifiesto el hecho de que las redes informales se extienden no sólo internamente, sino también más allá de la frontera de la empresa. Sattler, Schrader y Lüthje (2003) afirman además que la propensión a establecer redes informales de intercambio de informaciones varía en función del país, como resultado de distintos comportamientos, culturas y de diferentes estructuras sociales. Este tipo de perspectiva de carácter cultural, aunque muy interesante, todavía no ha sido trabajado de forma exhaustiva.

La importancia de los contactos informales también ha sido introducida en el debate sobre la geografía de la innovación. La proximidad entre empresas es un importante aspecto que puede determinar la capacidad innovadora de las organizaciones - la concentración geográfica facilita el flujo de información entre diferentes agentes (véase, por ejemplo, Russo, 1985 y Brusco, 1990). Una de las explicaciones se encuentra precisamente en el surgimiento de redes informales entre firmas como canales para la difusión de conocimiento (Dahl \& Pedersen, 2004).

Las colaboraciones y contactos informales, por lo tanto, juegan un importante y tal vez decisivo papel en la transmisión de información y en la creación de conocimiento en los individuos, al mismo tiempo que abre un interesante debate sobre la importancia del ámbito geográfico a la hora de cooperar y establecer redes de contactos. Mansfield (1985), sin embargo, apunta el lado negativo de los contactos informales, los cuales calificó de leakage of information. Él argumentó que la rápida difusión tecnológica a través de canales informales es una razón por la que las empresas tienen dificultad de apropiarse de los beneficios de la innovación. S. Schrader (199I) contra argumenta y afirma que los gestores no dejan dispersarse la información al alzar, sino que el comercio de información se hace conscientemente. Este autor además observa que la decisión de disipar información de la empresa a los demás está altamente influida por los costes y beneficios económicos de la transferencia. 


\section{Fuentes de datos y metodología}

\section{I Fuente de datos}

El análisis se realizará a partir de la in formación estadística que proporciona el Panel de Innovación Tecnológica (PITEC) para el año de 2004, resultado de un esfuerzo conjunto entre el Instituto Nacional de Estadística de España (INE), la Fundación Española para la Ciencia y la Tecnoᄀlogía (FECYT) y COTEC. Esta base de datos está construida a partir del Directorio Central de Empresas (DIRCE) y del Directorio de Empresas Posibles Investigadoras (DIRID) del INE. Este panel hace parte de la CIS española, y por lo tanto, los indicadores y variables de cooperación presentes en PITEC siguen las mismas directrices presentes en el Manual de Oslo.

El número de empresas de servicios recogidas en PITEC 2004 es de 3.546, de estas, 2.148 afirman ser innovadoras. Consideramos como empresa innovadora toda aquella empresa que afirma haber implementado algún tipo de innovación durante los últimos dos años, tal y como recomienda el Manual de Oslo (OCDE, 2005). Este concepto incluye, además de innovación tecnológica, cambios en el aspecto organizativo y de marketing. Las empresas innovadoras están clasificadas según la naturaleza de su actividad, en concordancia con la Clasificación Nacional de Actividades Económicas (CNAE).
La muestra del panel de 2003 se compone de dos grupos diferentes de las empresas: una muestra de empresas con 200 o más empleados y una muestra de empresas con gastos en I+D. La segunda edición del panel, en referencia a 2004, trató de cubrir una de las principales limitaciones que se encontraba en la primera edición, incorporando empresas con menos de 200 empleados, sin actividades de innovación, así como empresas con menos de 200 empleados cuyas actividades de investigación y desarrollo se han externalizado.

\subsection{Metodología}

Como forma de medir los contactos informales entre empresas y otros agentes en la innovación, hemos utilizado indicadores presentes en la Community Innovation Survey, que a su vez, ha sido diseñada siguiendo las directrices del Manual de Oslo (OCDE, 2005). No obstante, el cuestionario utilizado para esta encuesta europea carece de indicadores capaces de medir de forma clara el valor y la significatividad de los flujos informales de información.

Con respecto a las relaciones externas de la empresa, el cuestionario presenta únicamente, por un lado, una pregunta en la que la empresa debe valorar la significatividad de las fuentes de información y, por otro lado, otra pregunta sobre los agentes con quienes la empresa podría cooperar en proyectos de innovación. Sin embargo, en ninguna de las dos preguntas se permite valorar de forma explícita los contactos informales con clientes o proveedores, por ejemplo, sin la existencia de acuerdos formales como marco para este tipo de relación.

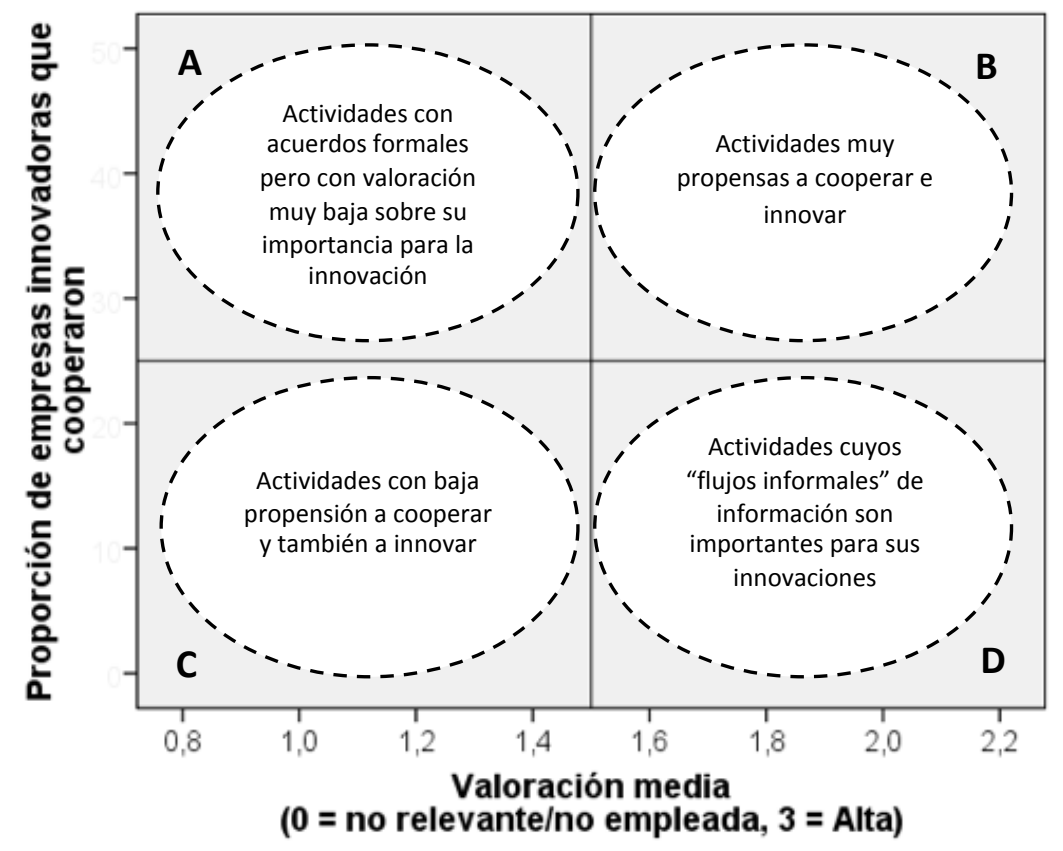

Ilustración I. Hipótesis para el análisis de los flujos informales de información. Fuente: Elaboración propia 
Para dar una solución a esta laguna, hemos propuesto un análisis comparativo entre esas dos variables, que se encuentra resumido en la ilustración I. La primera variable, representada en el eje vertical, se refiere a la proporción de empresas innovadoras que cooperaron por agente, medida en porcentajes. La segunda, representada en el eje horizontal, indica la valoración atribuida a cada uno de los agentes como fuente de información para la innovación. Esta variable está representada en una escala Likert cuyos valores fluctúan entre 0 y 3 , siendo 0 "nada relevante"/ "sin uso" y 3 "altamente relevante". La línea horizontal representa el promedio de los porcentajes de cooperación de todas las ramas de servicios, mientras que la vertical indica, en todos los casos, el punto de inflexión en la valoración $(I, 5)$ desde una perspectiva negativa, en la que la fuente no tiene mucha relevancia o simplemente uso $(<1,5)$, a una perspectiva positiva con valores muy o bastante importantes $(>1,5)$.

Por lo tanto, la hipótesis planteada para identificar el valor de los contactos informales para la innovación, es la siguiente: existirán posibles contactos informales en las ramas con porcentajes de empresas con cooperación con un determinado agente inferior al promedio del sector servicios, y que al mismo tiempo han valorado por encima del I,5 la relevancia de la información transmitida por parte de este mismo atente para la innovación. Esto implicaría que a pesar de una escasa práctica de actividades de cooperación formal, el conocimiento y la información es transmitida por vías alternativas, de tal forma que las empresas las consideran decisivas para sus procesos de innovación. En este sentido, la hipótesis del valor de los contactos informales estaría confirmada. El diagrama anterior trata de explicar esta hipótesis (ilustración I)'.

\section{Análisis empírico}

\section{I. El alcance de la innovación en los servicios}

Como introducción a este estudio empírico, empezaremos analizando el alcance de la innovación en el sector servicios para luego dar paso al estudio de los flujos informales de información. El objetivo de esta introducción es hacer patente la diversidad interna existente en el sector servicios en cuanto al comportamiento innovador. Esta evidencia servirá de base además para la interpretación de los resultados presentados en el apartado 4.2.

El sector servicios presenta patrones diferentes en cuanto a la proporción de empresas innovadoras por rama tal como se observa en la siguiente ilustración. El resultado es bastante esperado dado que muchos autores han tratado de resaltar las considerables diferencias entre el desempeño innovador de los diferentes servicios (Den Hertog \& Bilderbeek, 1999; Sundbo \& Gallouj, 2000; Tether \& Hipp, 2000; Hollenstein, 2003; Hipp \& Grupp, 2005, Miles, 2008; Trigo, 2009; Trigo \& Vence, 201I). La ilustración 2 permite observar un grupo de ramas de servicios intensivas en innovación y otro grupo muy poco intensivo. Las actividades de servicios a empresas son, desde luego, las más innovadoras con respecto al total, sumado a los servicios de "telecomunicaciones" e "intermediación financiera"2.

\footnotetext{
'Eso no excluye que no haya contactos informales en contextos donde existan cooperación formal. Las variables trabajadas simplemente impiden de discernir con precisión la naturaleza de los flujos de información cuando un sector es intensivo en cooperación y valora por encima del I,5 un determinado agente (cuadrante B).

${ }^{2}$ Cabe matizar que la elevada proporción de empresas innovadoras en PITEC, superior incluso a la de países como Alemania (véase Aschhoff et al., 2006) se debe en gran medida a la selección de empresas de la muestra. Recientes estudios empíricos revelan que España se sitúa por debajo de países como Italia, Portugal, Francia, Grecia, por no citar la propia Alemania y Luxemburgo en lo que se refiere a la innovación (véase Comisión Europea, 2006; Arundel, Kanerva, van Cruysen \& Hollanders, 2007). En realidad, España no sólo sostiene un índice sintético de innovación relativamente bajo para el conjunto del sector sino que se encuentra en el grupo de países que pierden terreno (loosing gound) en comparación con los demás. No obstante, para el caso en concreto de la intermediación financiera, España posee un índice sintético de innovación superior a muchos países de la UE (Kanerva, Hollanders \& Arundel, 2006).
}

ISSN: 07I8-2724. (http://www.jotmi.org) 


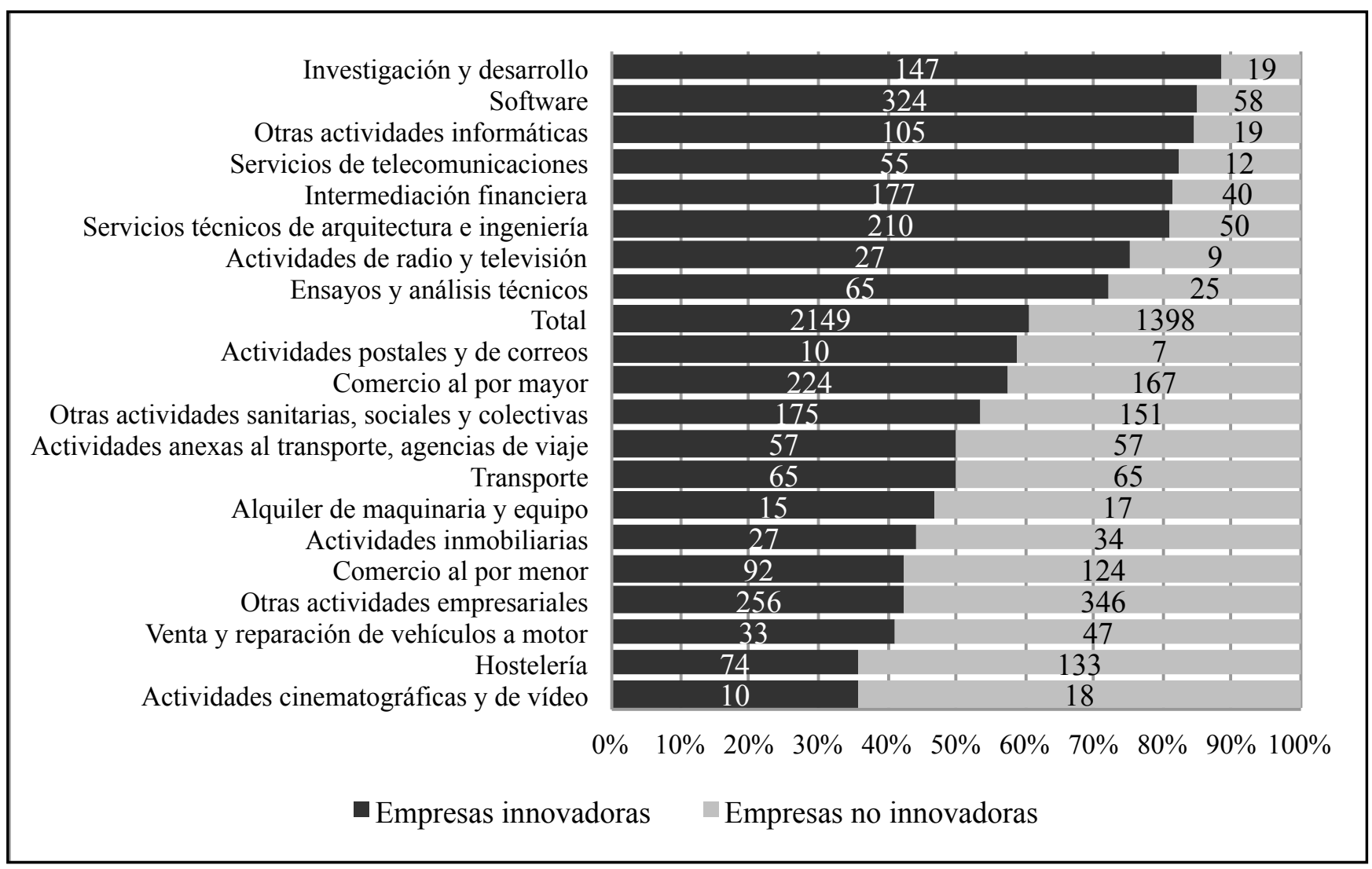

llustración 2. Proporción de empresas innovadoras, Servicios, España, PITEC 2004 (Número de empresas en cada barra). Fuente: Elaboración propia.

Sin embargo, ciertas actividades empresariales presentan un porcentaje bastante inferior en comparación con "otros servicios a empresas" (43\%) como por ejemplo actividades de fotografía, traducción, call centre, actividades industriales de limpieza, servicios de investigación y seguridad $y$, curiosamente, servicios de publicidad. Además de estos últimos servicios mencionados, un conjunto de actividades se encuentra también en la cola de la innovación compuesto por las actividades de "hostelería", "comercio al por menor", "servicios inmobiliarios", "actividades cinematográficas", "venta y reparación de vehículos a motor", entre otros. Tanto estos servicios como los anteriormente comentados son en su gran mayoría servicios personales y de distribución, respectivamente. Sus crecimientos no han sido tan significativos en la segunda mitad del pasado siglo, lo que confirma su escaso potencial innovador.

Diversos estudios empíricos realizados para otros países europeos han puesto en evidencia esta diversidad en cuanto a la performance innovadora de los servicios, lo que nos sirve como referencia para el caso español. Sin embargo, pocos han tratado de comparar dichos desempeños salvo escasas excepciones (Miles, 2008) ${ }^{3}$.

\footnotetext{
${ }^{3}$ Véase, por ejemplo, Tether et al. (2002) y Kanerva, Hollanders \& Arundel (2006), quienes presentan un índice sintético para la innovación en los servicios (Service Sector Innovation Index (SSII)), por sectores y por países.
} 


\subsection{Una aproximación empírica a los flujos informa- les en la producción y en la innovación en los servicios}

Tras comprobar la existencia de una diversidad en cuanto a la intensidad innovadoras de las actividades de servicios, hemos analizado el alcance le los contactos informales por rama económica siguiendo la metodología descripta en el apartado 3. Los resultados obtenidos demuestran primeramente que los contactos informales sólo están presentes en relaciones entre empresa y clientes, entre empresa y proveedor, así como dentro de la propia empresa. Dicho de otra forma, con excepción de las fuentes internas, los clientes y los proveedores, todas las fuentes de información han sido valoradas como "no

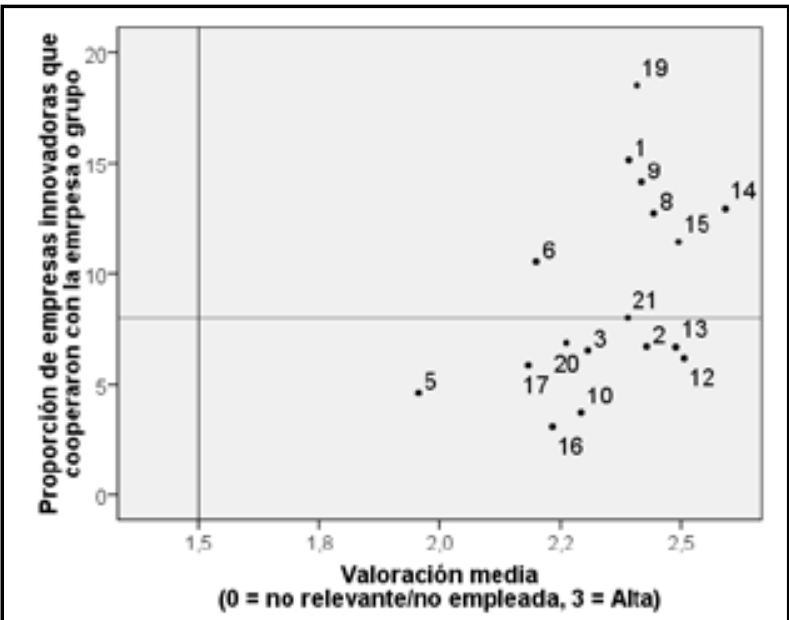

llustración 3

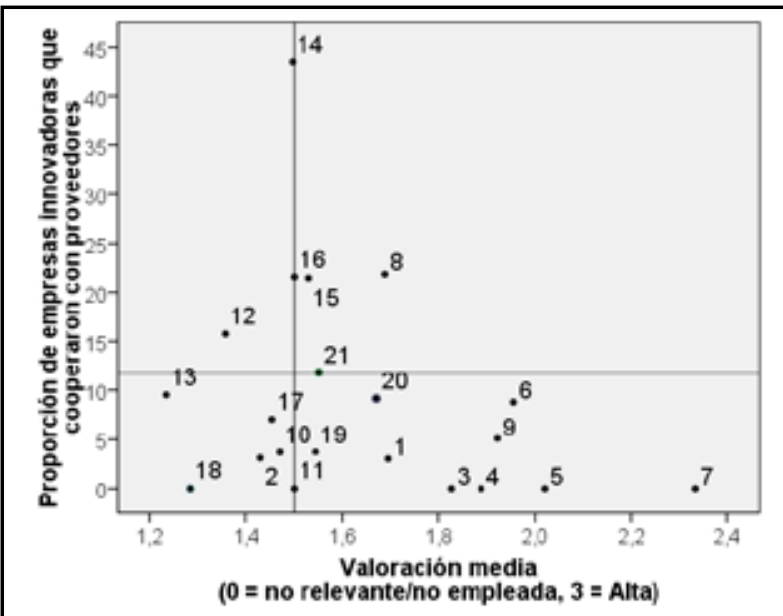

llustración 5 relevantes" o "poco importantes" (por debajo del 1.5 en la escala $0-3)^{4}$. Esa pauta en la valoración confirma los resultados encontrados por Tether (2002a) para una serie de países europeos ${ }^{5}$. Este resultado también coincide con las respuestas obtenidas en una encuesta propia llevada a cabo, entre otros, por Gallouj (2002). Las principales fuentes de información, conocimiento y competencias sobre producto, procesos organización y relaciones externas han sido los clientes, seguidos por la fuerza de trabajo interna y el personal contratado, a y a continuación los competidores.

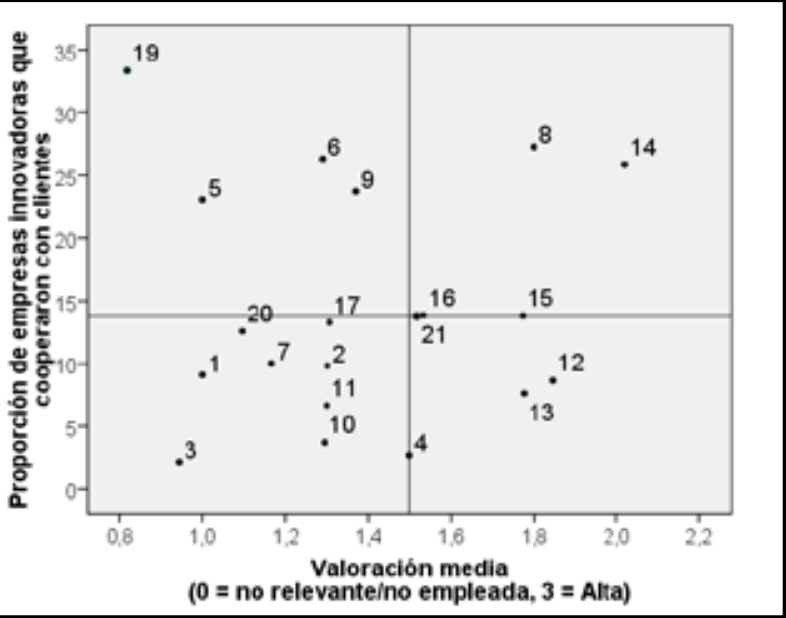

Ilustración 4

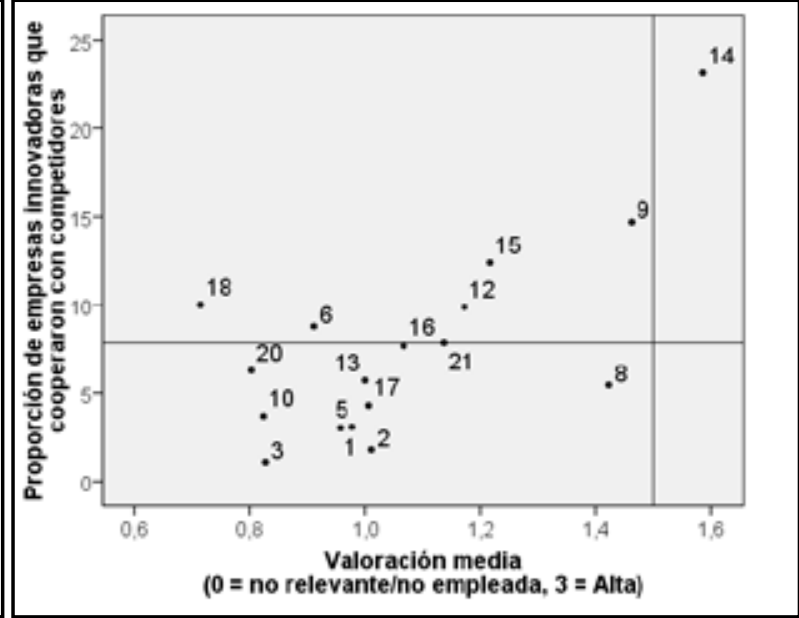

llustración 6

${ }^{4}$ Cabría matizar que la rama de investigación y desarrollo es la excepción pues presenta valoraciones bastante elevadas para todos los casos. ${ }^{5}$ Tether (2002a), en base a la segunda edición de la Encuesta Europea de Innovación CIS-2, identificó que las principales fuentes externas de información para la innovación en los servicios son: en primer lugar, los clientes, en segundo lugar, los proveedores y en tercer lugar, los competidores. Por otro lado, resultan de importancia marginal las universidades y los institutos de investigación. 


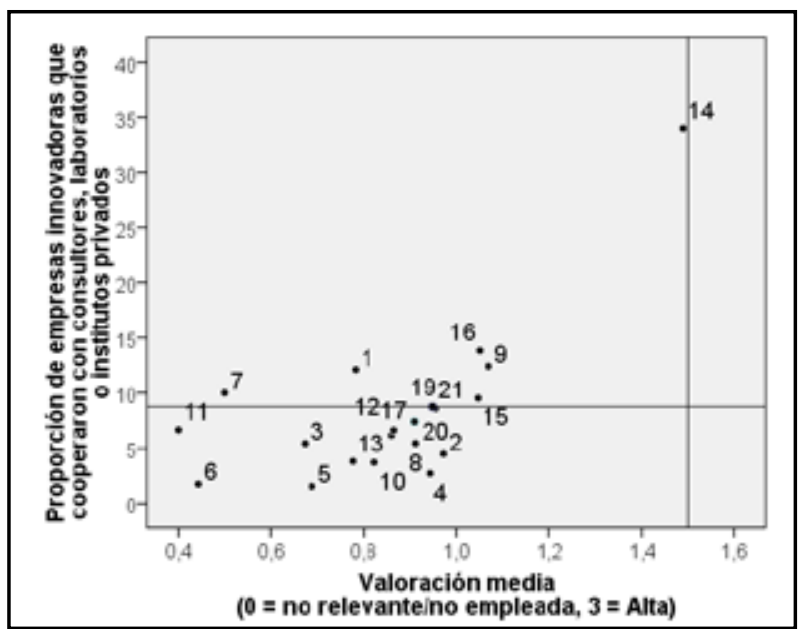

Ilustración 7

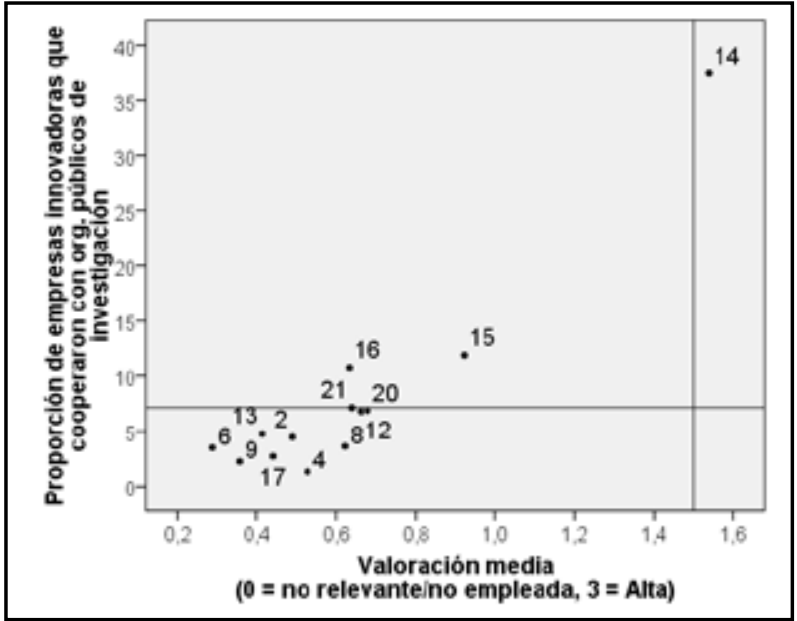

Ilustración 9

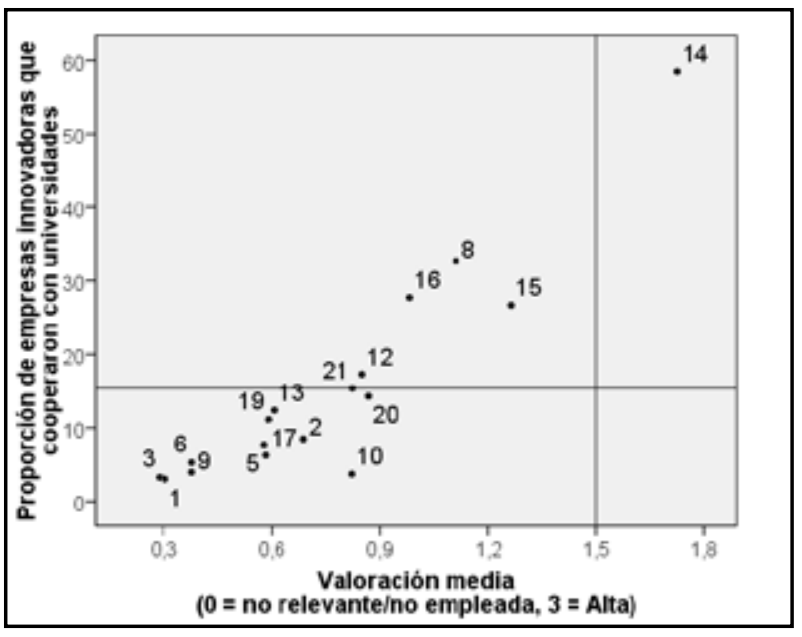

Ilustración 8

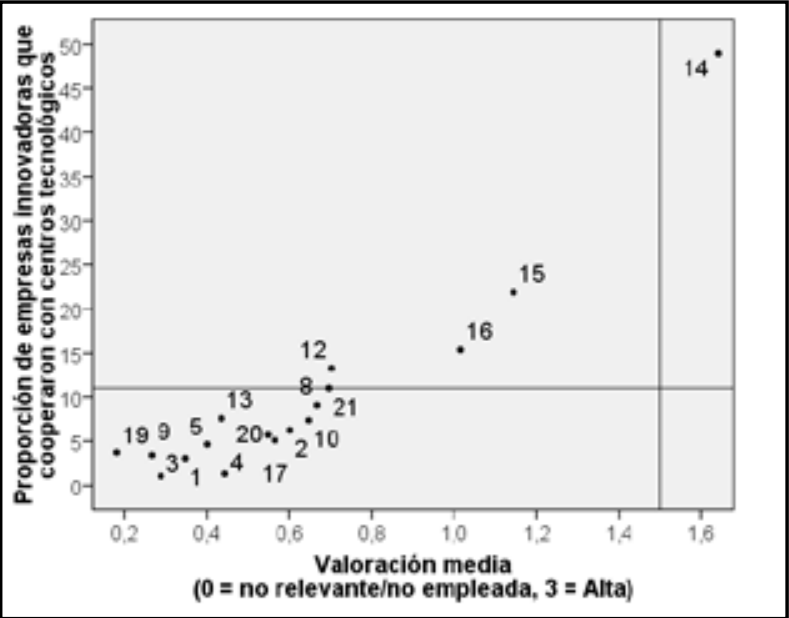

Ilustración 10

Ilustración 3-10. Proporción de empresas que cooperaron y valoración de la importancia de la fuente de información, por agente, Servicios, España. Fuente: Elaboración propia ${ }^{6}$.

\section{Leyenda:}

I Venta y reparación de vehículos a motor

2 Comercio al por mayor

3 Comercio al por menor

4 Hostelería

5 Transporte

6 Actividades anexas al transporte, agencias de viaje

7 Actividades postales y de correos

8 Servicios de telecomunicaciones

9 Intermediación financiera

10 Actividades inmobiliarias
II Alquiler de maquinaria y equipo

12 Software

13 Otras actividades informáticas

14 Investigación y desarrollo

I5 Servicios técnicos de arquitectura e ingeniería

16 Ensayos y análisis técnicos

17 Otras actividades empresariales

18 Actividades cinematográficas y de vídeo

19 Actividades de radio y televisión

20 Otras actividades sanitarias, sociales y colectivas

2I Total

${ }^{6}$ Los datos utilizados en el análisis son derivados del Panel de Innovación Tecnológica (PITEC) 2004. 
Resulta interesante observar que los casos donde existen aproximaciones informales se producen dentro de la cadena de producción de las empresas, o sea, con proveedores y clientes (relaciones verticales). Para el caso de los clientes, las actividades que se encuentran en el cuadrante D son "ensayo y análisis técnico", "software", el grupo de empresas pertenecientes a "otras actividades informáticas" y "hostelería". Se observa, por lo tanto, una mayor tendencia a los contactos informales con los clientes por parte de los servicios cualificados como specialised supplier, con excepción de la hostelería. En su gran mayoría se tratan de sectores muy intensivos en conocimiento e innovación como se ha podido observar en la ilustración 2. Esta agrupación se caracteriza básicamente por una relación muy cercana con el cliente, y por tanto por una consideración de este tipo de agente como importante fuente de información por encima de los demás.

Por otro lado, para el caso de los proveedores, los servicios presentes en el cuadrante $D$ son en su gran mayoría technology users. Las actividades que se incluyen en este grupo son "transporte y actividades anexas", "comercio al por menor" así como "transporte" y "actividades postales", entre otras (ilustración 5). En cierta manera, el perfil innovador y el comportamiento de estas ramas en cuanto a las variables seleccionadas se asemejan al patrón de innovación denominado supplier-dominated, identificado por Pavitt (1984) en su famosa tipología. Esas ramas se encuentran por debajo del promedio en cuanto a la proporción de empresas con I+D interna, así como del porcentaje de empresas innovadoras (con excepción, para este último caso, de la intermediación financiera y actividades de radio y televisión). Cabe también observar que en ambos casos, el promedio del sector servicios también se encuentra en este cuadrante, lo que en cierta forma refleja una tendencia positiva en este tipo de relación. Este perfil requiere una aproximación directa con los proveedores para innovar, en muchos casos, debido a que sus innovaciones ser basan en su gran mayoría en la aplicación y uso de tecnologías producidas por otras empresas.

Este resultado en el que los clientes y los proveedores se destacan por encima de los demás agentes parece razonable dado que todas las demás fuentes de información están representadas, en mayor o menor medida, por entidades en las que el acceso, y por lo tanto las relaciones entre individuos, requiere un cierto tipo de acuerdo o contrato. Esta afirmación ha sido sostenida también por $Ø$ stergaard (2007), quien ha probado que los flujos informales entre empresa y universidades, por ejemplo, se dan en mayor medida en empresas que afirman haber realizado algún tipo de cooperación con estos centros en concreto. Dicho de otra forma, los contactos informales surgen de relaciones establecidas previamente en el marco de acuerdos formales de colaboración. Esta observación ha sido comentada también en el apartado 3 referente a la metodología.

Otros resultados empíricos han demostrado que los flujos informales entre empresas se dan en mayor medida que entre empresa y organismos ligados a la ciencia y tecnología, como la universidad por ejemplo (Østergaard, 2007). Estos resultados pueden ser una evidencia más de la larga distancia entre el mundo académico-científico y la práctica empresarial. Otra explicación de las diferencias entre las valoraciones por agentes está en la naturaleza del conocimiento requerido por las empresas a la hora de innovar, lo que de cierta manera, se ve reflejado además en la extensión de los flujos tanto formales como informales de información y conocimiento.

Además, las empresas que valoran de forma elevada sus fuentes de información, así como las empresas con mayor tendencia a cooperar, pertenecen, en su gran mayoría, a sectores intensivos en tecnología ("investigación y desarrollo", "software", "telecomunicaciones", "ensayos y análisis técnicos", "servicios técnicos de arquitectura e ingeniería" y "otras actividades informáticas"), e intensivos en innovación (ilustración 2), aunque hay que destacar la elevada valoración de ciertos sectores sobre determinadas fuentes de información. De todas formas, esta evidencia refuerza las justificaciones en base a las diferencias entre el carácter técnico de las empresas y las valoraciones de los agentes externos.

A pesar de la originalidad de esta propuesta, este tipo de examen no tiene en consideración, el efecto positivo de los acuerdos formales de cooperación en relación con los flujos informales entre individuos que pueden ocurrir tanto durante como tras la finalización de un proyecto en común. Debido a esta laguna en la Community Innovation Survey, el autor de este artículo, en conjunto con los demás miembros del grupo ICEDE de la Universidad de Santiago de Compostela, España, ha añadido una pregunta específica en un cuestionario propio, elaborado y aplicado a empresas de diferentes sectores en la Comunidad Autónoma de Galicia, España, en el cual se diferencia los niveles de formalidad de las colaboraciones. Además de cuestionar la importancia de los proyectos conjuntos de 
innovación con otros agentes, se ha preguntado sobre la valoración de cualquier tipo de contacto o relación con clientes, proveedores, personal de otras empresas, etc., que derive en incorporación de nuevo conocimiento para la empresa. El resultado obtenido revela que, para todo el conjunto de empresas, la valoración de los contactos informales ha sido superior a la de los proyectos de innovación en cooperación. Las empresas de investigación y desarrollo son, una vez más, las que más han destacado la importancia de este tipo de relación, al mismo tiempo que también es el sector que más valora los contactos vía cooperación (véase para más detalles Trigo, 2009).

\section{Conclusiones}

El objetivo de este estudio ha consistido en presentar una propuesta de medición y análisis de los contactos informales llevados a cabo por empresas innovadoras con otros agentes del sistema como pueden ser los clientes, proveedores, universidades, empresas de la competencia, centros tecnológicos entre otros. La principal motivación para este esfuerzo se encuentra precisamente en la ausencia de indicadores que permitan examinar de forma explícita los flujos informales de información en el contexto de la innovación. Para alcanzar nuestro propósito, se ha realizado un cruce entre dos variables disponibles en la Community Innovation Survey (CIS). El primer indicador utilizado ha sido la proporción de empresas innovadoras que cooperaron, por agente, mientras que el segundo indicador aplicado se refiere a la valoración atribuida a cada uno de esos agentes sobre su importancia como fuente de información para la innovación.

El análisis empírico llevado a cabo en este estudio ha tratado de comparar el alcance de los contactos informales en diferentes actividades de servicios. Para ello, se han utilizado micro-datos del Panel de Innovación Tecnológica (PITEC) para España disponibles para el año 2004. Este panel está compuesto por empresas que participaron de la CIS española. La hipótesis planteada en la metodología de este análisis ha sido la siguiente: las ramas que han considerado una fuente de información determinada como muy o bastante importante, y que presentan a la vez un porcentaje reducido de empresas en cooperación formal con este agente específico, contemplan y valoran de forma eficaz los contactos informales.
Resulta interesante observar que los casos donde existen aproximaciones informales relevantes se producen dentro de la cadena de producción de las empresas, es decir, con proveedores y clientes (relaciones verticales). El análisis realizado permite identificar un grupo de ramas económicas que de cierta forma utiliza y valora positivamente los contactos informales con sus clientes. Este grupo de actividades económicas, perfilados en la literatura como specialised supplier, está compuesto, entre otros, por actividades de software, actividades informáticas, servicios técnicos de arquitectura e ingeniería, así como ensayos y análisis técnicos. Esta transcendencia incluye no sólo la propia prestación del servicio (recogida en la naturaleza de la actividad), sino que también se refiere a la relevancia de los consumidores como fuente de innovación, tal y como se describe en las variables seleccionadas.

Por otro lado, servicios caracterizados como technology users presentan en mayor medida flujos informales de información con sus proveedores. Esos servicios incluyen transporte y actividades anexas así como comercio al por menor, venta y reparación de vehículos a motor, y actividades postales.

Además de resaltar la importancia de los contactos informales a lo largo de las relaciones verticales, este análisis ha permitido identificar además flujos externos de información en sectores poco intensivos en tecnología. Resulta importante matizar que este tipo de manifestación difícilmente podría ser observado a través de indicadores de cooperación tradicionales, debido a que el tipo de alianza recogida en estos indicadores tiende a estar contextualizada en mayor medida en una esfera tecno-científica. Este es el caso de algunos estudios recientes (Trigo, 2009; Trigo \& Vence, 20II) que indican que aproximadamente el $59 \%$ de los servicios españoles innovadores no suelen llevar a cabo ningún tipo de cooperación a nivel formal, lo que no excluye necesariamente los flujos informales de información entre empresas y otros agentes.

Las colaboraciones y contactos informales, por lo tanto, juegan un papel importante y seguramente decisivo en la transmisión de información y en la creación de conocimiento en las empresas. Además, la investigación de estos intercambios de conocimiento abre un interesante debate sobre la importancia de la proximidad geográfica a la hora de cooperar y establecer redes de contactos, 
así como la relevancia del conocimiento tácito de los profesionales. El esfuerzo por elaborar una metodología de medición de los contactos informales, permite además afinar el estudio sectorial de la innovación y de los flujos de información, una vez que extiende el diagnóstico de las colaboraciones a una serie de actividades poco intensivas en tecnología y conocimiento, ignoradas durante mucho tiempo por la literatura especializada.

\section{Agradecimientos}

Quisiera expresar mi gratitud al Prof. Ian Miles del Manchester Institute of Innovation Research (University of Manchester, Reino Unido) por su apoyo y estímulo así como por sus sugerencias en las versiones preliminares de este trabajo. Agradezco también los comentarios de los miembros del Grupo de ICEDE de la Universidad de Santiago de Compostela, España. El autor agradece el apoyo financiero recibido por parte del Fondo Europeo de Desarrollo Regional (FEDER) así como de la Xunta de Galicia (Grupo de Referencia Competitiva 2008/04I y Proyecto 08SEC00820IPR).

\section{Referencias}

ALLEN, J., James, A., \& Gamlen, P. (2007). Formal versus informal knowledge networks: challenges for R\&D management, R\&D Management, 37, 3, pp. 179-196.

ANDERSEN, B., Metcalfe, J. S., y Tether, B. (2000). Distributed Innovation Systems and Instituted Economic Process, in Metcalfe J. S. y Miles I. (eds.) Innovation systems in the service economy. Measurements and case study analysis. Boston: Kluwer, pp. 15-42.

ANTONELLI, C. (1998). Localized Technological Change, New Information Technology and the Knowledge-Based Economy: The European Evidence, Journal of Evolutionary Economics, 8, pp. 177-198.

ANTONELLI, C. (2005). Models of Knowledge and Systems of Governance, Journal of Institutional Economics, I, I, pp. 5I-73.

ARUNDEL, A., Kanerva, M., VAN Cruysen, A. \& Hollanders, H. (2007). Innovation Statistics for the European Service Sector. INNO Metrics Report, Brussels, European Commission, DG Enterprise.
ASCHHOFF, B. et al. (2006). Innovation in Germany: Results of the German Innovation Survey 2005, Mannheim.

BRUSCO, S. (1990). The idea of the industrial district: its genesis. In: Pyke, F., Becattini, G. \& Sengenberger, W. (eds.), Industrial Districts and Inter-Firm Cooperation in Italy. International Institute for Labour Studies, Geneva, pp. 10-19.

CHANDLER, A. (1962). Strategy and Structure: chapters in the history of the industrial enterprise. Cambridge, MA; London: MIT Press.

CHESBROUGH, H. (2003). Open Innovation: The New Imperative for Creating and Profiting from Technology. Boston, Mass.: Harvard Business School Press.

CHESBROUGH, H. et al. (eds.) (2006). Open Innovation: Researching a New Paradigm. Oxford: Oxford University Press.

CHRISTENSEN, J. L. \& Lundvall, B.-Å. (eds.) (2004). Product Innovation, Interactive Learning and Economic Performance. Amsterdam: Elsevier.

COMISIÓN EUROPEA (2006). European Innovation Scoreboard 2006 - Comparative Analysis of Innovation Performance. Pro Inno Europe. Inno Metrics. MERIT - Maastricht Economic and social Research and training centre on Innovation and Technology.

COOMBS, R., Harvey, M., \& Tether, B. (2003). Analysing distributed processes of provision and innovation, Industrial and Corporate Change, I2, 6, pp. II25-II55.

CROSS, R. \& Parker, A. (2004). The Hidden Power of Social Networks. Boston: Harvard Business School Press.

CZARNITZKY, D. \& Fier, A. (2003). Publicly funded R\&D collaborations and patent outcome in Germany, ZEW Working Paper, N. 03-24, Center for European Economic Research, Mannheim.

DAHL, M. S. \& Pedersen, C.Ø.R. (2004). Knowledge flows through informal contacts in industrial clusters: myth or reality?, Research Policy, 33, pp. 1673-1686.

DEN HERTOG, P. \& Bilderbeek, R. (1999). Conceptualising service innovation and service innovation patterns, Research Programme on Innovation in Services (SIID) for the Ministry of Economic Affairs, Dialogic, Utrecht. 
DOUGHERTY, V. (1999). "Knowledge is about people, not databases", Industrial and Commercial Training, 3I, 7, pp. 262-266.

EDQUIST, C. (ed.) (1997). Systems of Innovations. London: Pinter Publishers.

FREEMAN, C. (1987a). Factor Substitution and the Instability of Growth, paper prepared for a Symposium at the Institute of Statistical Research, Tokyo, Japan on 23 and 24 September 1987.

FREEMAN, C. (1987b). Technology and Economic Performance: Lessons from Japan. London: Pinter Publishers. GALLOUJ, F. (2002). Innovation in the service economy: the newwealth of nations. Cheltenham:EdwardElgarPublishing.

GALLOUJ, F. (2007). Economia da Inovação: um Balanço dos Debates Recentes. In: Bernades, R. \& Andreassi, T. (eds.), Inovação em Serviços Intensivos em Conhecimento. São Paulo: Editora Saraiva, pp. 3-28.

GEMÜNDEN, H.G., Heydebreck, P. \& Herden, R. (1992). Technological Interweavement: A Means of Achieving Innovation Success, R\&D Management, 22, 4, pp. 359- 376.

GIBBONS, M. et al. (1994). The New Production of Knowledge: The Dynamics of Science and Research in Contemporary Societies. London: Sage Publications.

HAYEK, F. A. (1945). The Use of Knowledge in Society, American Economic Review, XXXV, 4, pp. 519-530.

HIPP, C. \& Grupp, H. (2005). Innovation in the Service sector: The demand for service-specific innovation measurement concepts and typologies, Research Policy, 34, pp. 517-535.

HOLLENSTEIN, H. (2003). Innovation modes in the Swiss service sector: a cluster analysis based on firms-level data, Research Policy, 32, pp. 845-863.

KANERVA, M, Hollanders, H. \& Arundel, A. (2006). Can we measure and compare innovation in services? European Trend Chart on Innovation report, European Commission, Brussels, 17.

LUNDVALL, B.-Å. (ed.) (1992). National System of Innovation: Towards a Theory of Innovation and Interactive Learning. London: Pinter Publishers.
MANSFIELD, E. (1985). "How Rapidly Does New Industrial Technology Leak Out?”, Journal of Industrial Economics, 34, pp. 217-223.

MILES, I, (2008). Patterns of innovation in service industries, IBM Systems Journal, 47, I, Pp. II5-I28.

MILES, I. \& TETHER, B. (2003). "Innovation in the Service Economy”, IPTS Report, 7I, pp. 45-5I.

MILES, I. (1993). "Services in the new industrial economy", Futures, 25, 6, pp. 653-672.

MILES, I. (1994). "Innovation in Services" in Dodgson, M.\& Rothwell, R. (eds.), The Handbook of Industrial Innovation. Cheltenham: Edward Elgar Publishing, pp. 243-256.

NELSON, R. R. (ed.) (1993). National innovation systems: a comparative analysis. New York: Oxford University Press.

OECD (2005). Oslo Manual - Proposed Guidelines for Collecting and Interpreting Technological Innovation Data. Paris.

OERLEMANS, L., Meeus, M. AND Boekema, F. (1998). Do networks matter for innovation? The usefulness of the economic network approach in analysing innovation, Tijdschrift voor Economische en Sociale Geografie, 89, 3, Pp. 298-309.

ØSTERGAARD, C. R. (2007). Knowledge flows through social networks in a cluster: comparing university and industry links, Paper for the 5th International EMAEE Conference May 2007. Manchester Metropolitan University, Manchester, Reino Unido.

PAVITT, K. (1984). Sectoral patterns of technical change: towards a taxonomy and a theory, Research Policy, 13, 6, pp. 343-373.

ROTHWELL, R. \& Robertson, A. B. (1973). The role of communication in technological innovation, Research Policy, 2, pp. 204-225.

ROTHWELL, R. (1992). Successful Industrial Innovation Critical Factors for the 1990s, R\&D Management, 22, 3, pp. 22I-239.

ROTHWELL, R. (1994). Towards the fifth-generation innovation process, International Marketing Review, II, I, pp. 7-3I. 
RUSSO, M. (1985). Technical change and the industrial district: the role of interfirm relations in the growth and transformation of ceramic tile production in Italy, Research Policy, I4, pp. 329-343.

SATTLER, H. Schrader, S. \& Lüthje, C. (2003). Informal cooperation in the US and Germany: cooperative managerial capitalism vs. competitive managerial capitalism in interfirm information trading, International Business Review, 12, 3, pp. 273-295.

SINGELMANN,J.(1978). TheSectoralTransformation of the Labor Force in Seven Industrialized Countries, 1920-1970, The American Journal of Sociology, 83, 5, pp. 1224-1234.

SUNDBO, J. \& GALLOUJ, F., (2000). Innovation as a Loosely Coupled System in Services. In: Metcalfe J. S. \& Miles I, (eds.), Innovation systems in the service economy. Measurements and case study analysis. Boston: Kluwer, pp. 43-68.

TAMINIAU, Y., Smit, W., de Lange, A. (2009). Innovation in management consulting firms through informal knowledge sharing, Journal of Knowledge Management, I3(I): 42-55.

TETHER, B \&, Hipp, C., (2000). Competition and innovation amongst knowledge-intensive and other service firms: evidence from Germany. In: Andersen, B., Howells, J., Hull, R., Miles, I. \& Roberts, J, (eds.), Knowledge and Innovation in the New Service Economy. Cheltenham: Edward Elgar Publishing, pp. 49-67.

TETHER, B. (2002a). The sources and aims of innovation in services: variety between and within sectors, CRIC Discussion Paper No 55, Manchester.

TETHER, B. (2002b). Who co-operates for innovation, and why. An empirical analysis, Research Policy, 3I, PP. 947-967.

TETHER, B. et al. (2002). Innovation in the Service Sector: Analysis of Data Collectedunder the Community Innovation Survey (CIS-2), CRIC Working Paper No II, Manchester.

Tödtling, F. et al (2009). Do different types of innovation rely on specific kinds of knowledge interactions?, Technovation, volume 29 , I, pp. 59-7I.
TRIGO, A. \& Vence, X. (20II). Too many lonely innovators. The cooperation behavior of Spanish innovating service enterprises, Research Policy (Forthcoming).

TRIGO, A. (2009). La naturaleza y la dinámica de los flujos de información en innovación. Un análisis del sector servicios en España. Santiago de Compostela: Universidad de Santiago de Compostela.

VEGA-JURADO, J., Gutiérrez-Gracia, A. \& FernándezDe-Lucio, I. (2009). Does external knowledge sourcing matter for innovation? Evidence from the Spanish manufacturing industry, Industrial and Corporate Change, Volume 18, Number 4, pp. 637-670. 
J. Technol. Manag. Innov. 20II,Volume 6, Issue 2 